\title{
Ingénition : Une méthode stratégique pour la génération de la connaissance
}

Stéphane Brunel, Marc Zolghadri, Philippe Girard, IMS-LAPS, Université Bordeaux 1, 351

Cours de la Libération, 33405 Talence Cedex - France - Tel/Fax: +33(5) 40002405 / 6644, Courriel : $\underline{\text { stephane.brunel@ims-bordeaux.fr }}$

Stéphane Brunel est professeur de Technologie à l'IUFM d'Aquitaine, Ecole interne de l'Université Bordeaux 4 et doctorant au laboratoire IMS Equipe ICO de l'Université de Bordeaux 1. Il est Directeur Adjoint de l'IUFM d'Aquitaine, responsable de la filière Technologique pour les professeurs stagiaires.

Marc Zolghadri est Maître de Conférences à l'IUFM d'Aquitaine, Ecole interne de l'Université Bordeaux 4 et membre du laboratoire IMS Equipe ICO de l’Université de Bordeaux 1.

Philippe Girard est Professeur des Universités, Directeur de l'IUFM d'Aquitaine, Ecole interne de l'Université Bordeaux 4. Il est le responsable de l'Equipe ICO-Ingénierie de la Conception du Laboratoire IMS de l'Université de Bordeaux 1.

Résumé : Dans cet article, nous présentons, des propositions organisées autour d'une nouvelle méthode qui devrait permettre à des entreprises de mieux se positionner stratégiquement sur le marché très concurrentiel des produits destinés à la transmission des connaissances. Ces propositions tournent autour de l'idée que toutes les connaissances générées au cours de la conception de produit participent à l'élaboration de produits plus performants destinés à la transmission des connaissances. Pour ce faire, on propose un cadre générique contenant un macro modèle et un ensemble de micro-modèles. Tous sont nécessaires pour analyser la situation de la connaissance de l'entreprise et pour concevoir une sorte de «roadmap». Cette carte stratégique issue de l'expérience mutualisée des différents services de l'entreprise se construit sur un principe de double flux. En même temps que je conçois, je génère la connaissance qui me servira à former et à apprendre sur le produit lui-même. La conception, la transmission et l'apprentissage grâce au produit ainsi généré, participeront à la formation des employés eux-mêmes et répondront aux besoins des nouveaux clients.

Mots clés : Conception, Ingénition, Connaissance, Apprentissage, Stratégie. 


\section{INTRODUCTION}

La connaissance est devenue un enjeu majeur au sein des entreprises pour plusieurs raisons. Si au cours des dernières décennies, la maîtrise technologique et les moyens de production étaient les seuls enjeux stratégiques, on voit depuis quelques années que cette différenciation concurrentielle ne suffit plus. L'idée principale de notre travail est que l'ensemble des personnels-acteurs de l'entreprise doit être engagé dans un processus de création et de gestion de la connaissance. Non seulement les personnels des différents services produisent de la connaissance mais ils sont amenés aussi à se former tout au long de leur vie donc à s'approprier de la connaissance. Ils la transformeront en compétences au cours de la mise en œuvre de celles ci dans leur métier. Cette idée n'est pas neuve, Porter l'a très bien décrite [20]. Les chercheurs de la communauté de la connaissance abordent de multiples façons cette problématique. On voit bien que c'est véritablement un enjeu crucial. Ces investigations semblent se déplacer aujourd'hui vers une conjonction de domaines et de facteurs et d'inducteurs différents. La Figure 1 montre l'intersection de trois domaines : théories de la conception, théories de l'apprentissage et théories de la connaissance. Les théories de la connaissance tiennent compte de la gestion de celles-ci mais intègrent rarement les concepts issus des théories de l'apprentissage. Les modèles considèrent les personnels-acteurs de l'entreprise comme des personnes hautement qualifiées et entraînées qui n'ont pas besoin de formations continues complémentaires. On sait que les connaissances acquises en entrée ne sont pas du tout les mêmes que les connaissances de l'expérience pour ne prendre qu'une partie du problème. Le premier axiome de notre recherche est qu'il est nécessaire d'associer divers éléments de la connaissance produite au cours des phases de la vie du produit.

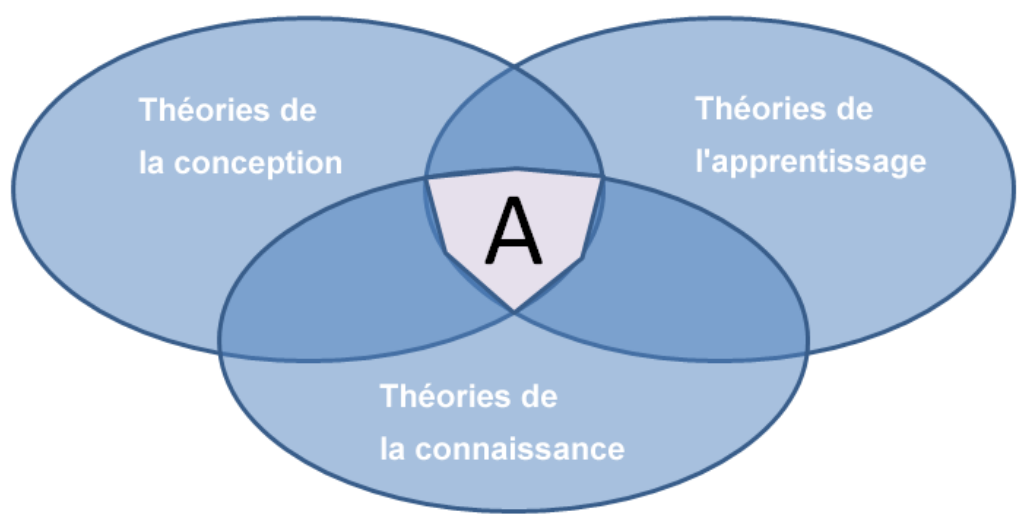


Figure 1 - Intersection de trois théories

La conjonction des stratégies de formation et de la gestion des connaissances offre un caractère dynamique à cette connaissance générée. N'importe quel élément de la connaissance une fois produit, analysé, structuré et enregistré devient un catalyseur qui devra amplifier et structurer les éléments des nouvelles connaissances apportés. C'est un procédé dynamique en expansion appelé « ingénition». Le travail entrepris cherche à fournir un cadre formel pour ce processus. Les macro et micro modèles permettront d'envisager les interactions entre les composants du processus de génération des connaissances et permettront, par exemple, la définition d'une planification stratégique de la formation.

L'article est structuré de la façon suivante: Le chapitre 2 élabore un bref état de l'art tout en recensant les théories qui ont impactées fortement le futur modèle. Le « processus d'ingénition » est défini avec précision dans le chapitre 3. Il comprend également la description du macro modèle. Les formalismes plus détaillés d'une modélisation sont proposés dans le chapitre 4. Nous terminons avec des pistes de réflexion et des possibilités d'ouverture sur des travaux futurs.

\section{ETAT DE L'ART}

Notre propos se situe sans ambigüité dans le domaine de la conception. C'est pour cela que notre modèle va chercher ses sources dans les travaux précédents qui son analysés ici. Nous nous sommes inspirés fortement des travaux de Merlo [15].

\subsection{LES METHODES DE CONCEPTION}

Dixon [5], Evbuomwan [9] ou Perrin [19] recensent l'ensemble des travaux sur la conception et les classent en trois catégories : les modèles prescriptifs, les modèles descriptifs (ou cognitifs), les modèles «computationnels». Ici s'opposent deux visions : celle du processus centré sur les transformations et les flux d'informations et celle centrée sur l'homme qui tente indirectement de comprendre comment fonctionnent ses capacités d'analyse, d'abstraction et de création. Les modèles computationnels font la synthèse des deux visions.

\subsubsection{Modèles prescriptifs de la conception}

Selon Pahl \& Beitz [17], le modèle décrivant la conception comme une «succession hiérarchique de phases » correspond à une vision macroscopique. Il comporte quatre phases : la définition du cahier des charges ou problème à résoudre, la conception de principes (« conceptual 
design »), la conception d'ensemble («embodiment design») et enfin la conception de détail (« detail design »). Plusieurs chercheurs ont montré qu'à l'intérieur de chaque phase, les acteurs suivent un «cycle élémentaire et itératif» pour résoudre un problème donné. Blessing [1], Roozenburg et Eekels [21], évoquent de manière différente des cycles répétitifs et itératifs. Ces modèles sont trop généraux. La solution envisagée est le résultat d'un compromis entre différents points de vue, il s'agit d'un «phénomène émergent d'auto-organisation». Perrin [18] considère que durant ces périodes de coopération, les acteurs mettent en œuvre un processus d'apprentissage s'appuyant sur des ressources cognitives, organisationnelles et institutionnelles permettant d'obtenir ce compromis. Il est donc important de permettre aux acteurs de capitaliser leurs expériences pour développer leurs compétences, ce qui correspond à l'identification d'objectifs de conduite à long terme, dépassant la durée de vie d'un projet.

\subsubsection{Modèles descriptifs de la conception}

Cross [3] met en avant un aspect essentiel pour nous, c'est l'aspect du «processus cognitif ». Dixon [5] étudie la façon dont les acteurs acquièrent des compétences et les mettent en jeu dans un processus de conception. Schön [22] assimile l'activité cognitive du concepteur à une « forme de communication et de conversation ».

\subsection{METHODES ISSUES DE L'INGENIERIE DES CONNAISSANCES}

Nous pouvons identifier trois approches distinctes : une approche de modélisation descendante, une approche ascendante et une approche coopérative.

\subsubsection{Approche descendante}

Les méthodes, MKSM puis MASK [7], développées par Ermine [6] permettent à un expert l'analyse et la formalisation des connaissances pour constituer une base de connaissances ainsi que la capitalisation de savoir ou de savoir-faire, la structuration de corpus documentaires ou scientifiques, ou encore la diffusion de connaissances. Cette méthode repose sur deux hypothèses préalables qui constituent un outil d'exploration du système de connaissances : le macroscope. Elle met en avant, l'hypothèse sémiotique: la syntaxe, la sémantique et la pragmatique ; l'hypothèse systémique : c'est un système de connaissances au sens de Le Moigne [12]. 


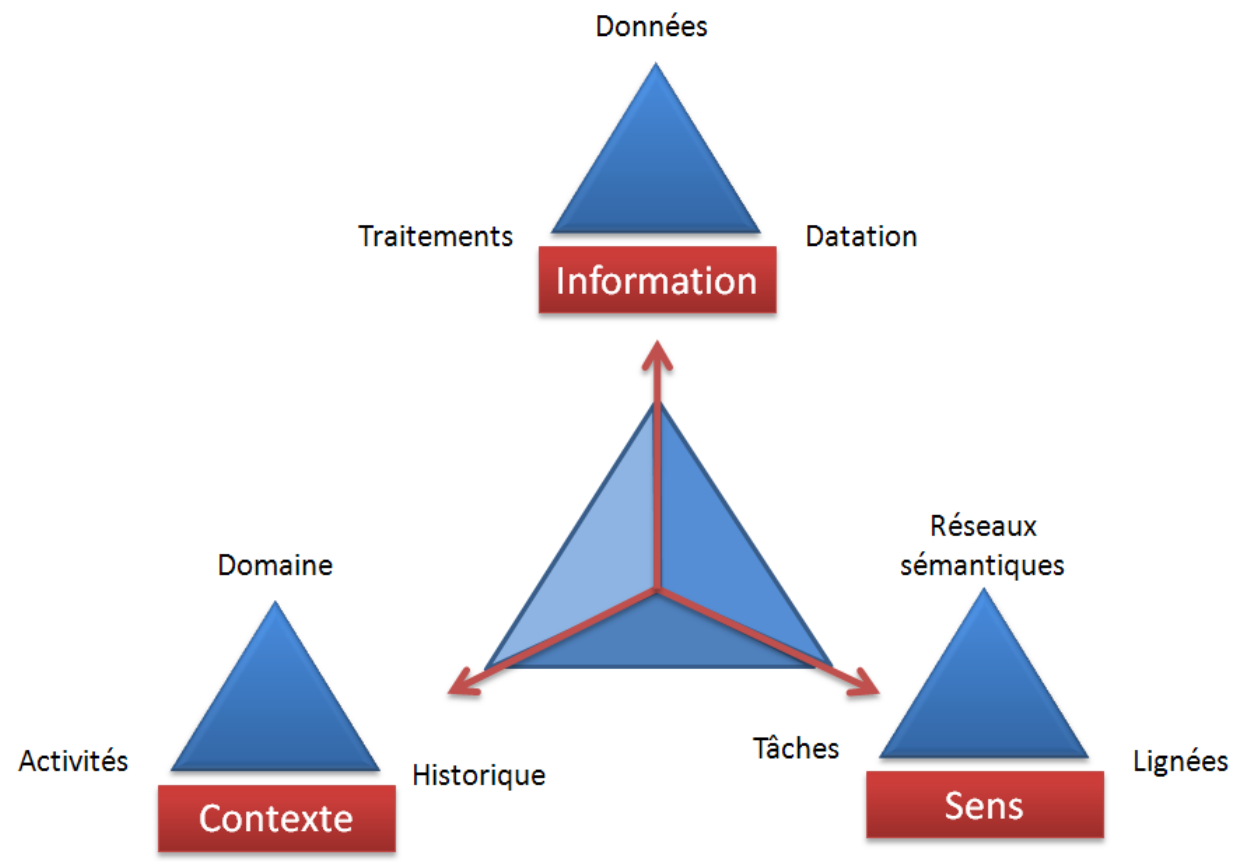

Figure 2 : Macroscope de la connaissance [7]

A partir de ces hypothèses MKSM formule une démarche de capitalisation des connaissances qui intègre six de ces neuf points de vue. Elle comporte cinq phases de modélisation : le modèle du «système de référence» de Le Moigne [13], le modèle du «domaine» et le modèle «d'activité », le modèle des « concepts » et le modèle des «tâches » [8]. MASK [14] prolonge MKSM et intègre un modèle de "l'historique », un modèle des «lignées », un mécanisme de «datation». Ces démarches sont particulièrement intéressantes dans le cadre de notre problématique en cimentant notre propre contribution.

\subsubsection{Approche ascendante}

Les approches de ce type s'appuient sur des méthodes de recueil des connaissances et font appel à des outils d'interprétation permettant la modélisation à posteriori. Schreiber [23, 24], à partir des méthodes KADS ou CommonKads ainsi que Caulier [2], Simon [25], Glaser [11], Wielinga [26] exploitent cette approche ; CommonKADS [4] étend KADS. Il n'est pas dans notre propos de détailler l'ensemble de ces méthodes dans cet article.

Ces exemples tous aussi fertiles les uns que les autres, donnent une assise complémentaire à notre proposition future. Nous ne proposons en aucune manière quelque chose de différent mais plutôt une stratégie organisatrice intégrant ces différentes approches. 


\section{INGENITION : UN PROCESSUS DE CONCEPTION DES PRODUITS POUR}

\section{LA TRANSMISSION ET/OU L’APPRENTISSAGE DES CONNAISSANCES.}

Le processus d'ingénition est basé sur un cycle mettant en commun les connaissances, les compétences et les acteurs au sein de l'entreprise (voir le Figure 4). Pendant la toute première opération de ce procédé, un macro modèle est défini (la grille en haut de la Figure 4) afin de déterminer les orientations stratégiques de l'entreprise en termes de génération des connaissances des employés ainsi que de déterminer de façon plus précise les besoins des futurs clients. Les détails de cette grille sont fournis dans le prochain paragraphe. Les situations d'apprentissage sont créées grâce à des collaborations existantes; elles mêmes supportées par des «produits orientés apprentissage ». Nous appelons ces produits : "produits orientés didactique".

Ces situations sont modélisées et nommées par le graphe des connaissances « AS IS » (Figure 3). Ces graphes sont obtenus après observation des actions et des réactions des stagiaires et de leurs formateurs pendant le processus d'apprentissage. Par exemple, de jeunes ingénieurs dans un bureau d'étude prennent des informations concernant les procédures diverses auprès des plus anciens.

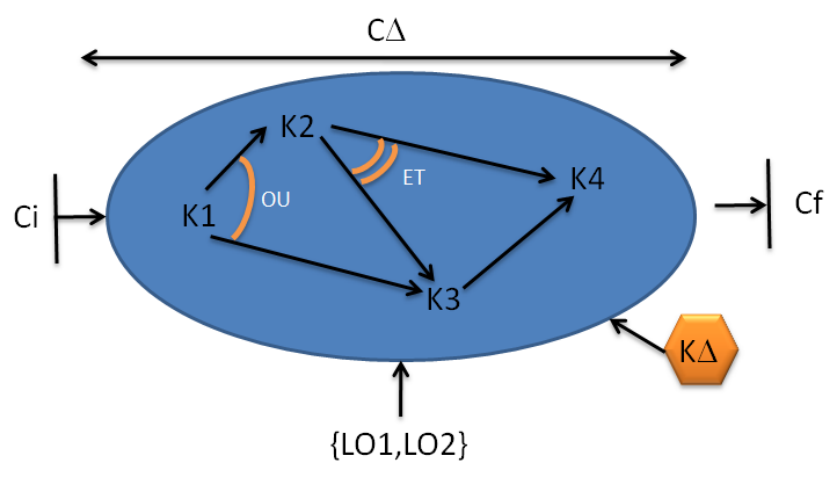

Figure 3 : Exemple de Graphe des connaissances 


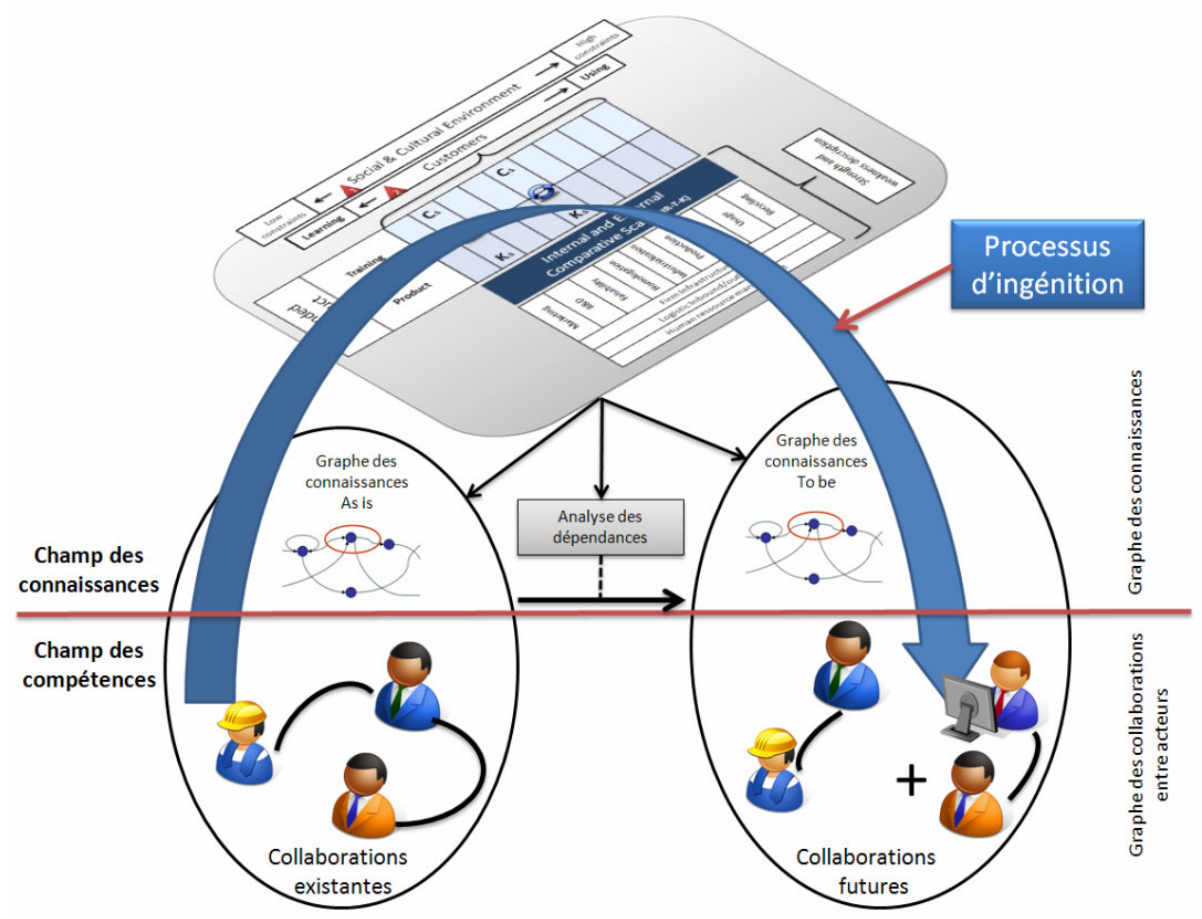

Figure 4 : Modèle Général du Processus d’Ingénition

Après avoir analysé ces graphes, concernant les diverses connaissances et compétences susceptibles d'atteindre l'objectif visé, plusieurs graphes appelés «Graphes des connaissances (To $\mathrm{Be}$ ) » peuvent être établis. Une fois évalués et analysés, les graphes les plus appropriés sont choisis et ils seront utilisés comme cadre principal à partir duquel des contraintes particulières pour le modèle devront être extraites. D'ailleurs, de ce nouveau graphe, des protocoles concernant des sessions de formation peuvent être recensés afin de satisfaire au mieux les exigences des futurs clients. La méthodologie complète du «processus d'ingénition » n'est pas décrite ici. Nous décrivons seulement ses deux outils principaux.

\subsection{LE MACRO MODELE DU PROCESSUS D'INGENITION}

Quand un responsable de projet au sens de Nonaka [16] conduit sa mission, il devrait avoir un point de vue (horizontal). En étendant cette notion à tout le cycle de vie de produit, il peut voir que le processus d'apprentissage croise systématiquement les phases du cycle de vie du produit. Cela signifie qu'il y a un processus d'apprentissage parallèle à tout le cycle de vie. Cette vision n'est parfois pas possible pour les gestionnaires de système qui ont très souvent un point de vue fonctionnel (ou vertical) d'une partie donnée du système. Ceci signifie que pour un projet donné au cours de son développement, il est nécessaire de représenter toute la stratégie globale pour 
identifier les processus d'apprentissage verticaux et horizontaux. Nous utilisons un macro modèle pour représenter ces deux vues complémentaires (Figure 5). Une fois établi, ce modèle rend l'identification, de la plupart des éléments indispensables à la conception du produit, possible.

L'idée de ce chapitre est d'établir un réseau d'analyse qui va aider les décideurs pour formaliser leur décisions stratégiques. Nous cherchons à comprendre comment la connaissance croît, ainsi que les savoir-faire afin de recenser des méthodes pour la capitalisation de la connaissance et la capitalisation de ces savoir-faire au cours de la conception du produit. En fait, de nombreuses décisions devront être prises en se basant sur les caractéristiques issues de ce modèle des connaissances. En conséquence, le réseau doit permettre l'expression de divers niveaux de décision de conception par l'élaboration d'une granularité différente. Au niveau stratégique, une des finalités est d'avoir un point de vue global pour les objectifs de formation envisagés au sein même de l'entreprise. Les décideurs discernent mieux comment les divers facteurs (internes et externes) peuvent influencer le processus, la production et l'organisation de la conception. Nous proposons des éléments d'aide à cette compréhension par cette grille. Son rôle principal est d'aider à la formulation des décisions concernant des formations nécessaires et/ou proposées. Nous allons la décrire pas à pas. Cette grille, à savoir «processus d'ingénition», est décomposée en cinq composants principaux (Figure 5):

1. Le contexte social, culturel et environnemental ;

2. L'utilisation du futur produit par le client ;

3. La génération de la connaissance au cours du cycle de vie du produit étendu ;

4. L'échelle comparative des ressources internes et externes ;

5. La chaîne de valeur par la décomposition des activités de l'entreprise pour lesquelles nous analysons la génération des connaissances.

Cette grille contient deux parties distinctes : 1) Le contexte permettant la description des contraintes de l'environnement. 2) Le fonctionnement des différents services, décrivant les forces et les faiblesses de l'entreprise au cours de la durée de vie du produit en faisant la comparaison des ressources humaines et des connaissances des acteurs. Initialement, cette grille permet de décrire la position réelle (as is) pour la société dans le cadre du lancement d'un nouveau projet. Cette analyse permet aux décideurs de recenser les conditions d'investissement dans de nouveaux outils par exemple. Elle détermine une stratégie d'acquisition de moyens. Nous proposons la 
formulation de cette stratégie en trois points : Formulation des besoins, la mise en valeur des interdépendances entre les diverses acquisitions et la planification de la formation dans la période nécessaire. L'exécution de cette stratégie devrait permettre la réalisation de l'objectif prédéfini (TO BE).

\subsubsection{Le contexte social, culturel et environnemental}

Un produit est conçu ou bien pour son utilisation ou bien dans un but d'apprentissage. Ses caractéristiques (fonctionnelles, structurelles et comportementales) ne sont donc pas forcément identiques. Évidemment, il y a une échelle continue allant des produits d'usage à ceux conçus dans un but d'apprentissage exclusif. Les contraintes sociales et culturelles doivent être également prises en compte. En schématisant ces contraintes par la position d'un curseur sur une échelle continue, le contexte socioculturel et l'environnement du produit est alors défini. Les audits des experts de l'entreprise permettront la définition de la position relative de ce curseur. Nous pourrions envisager d'utiliser les retours d'expérience pour étayer le positionnement de ce curseur.

\subsubsection{L'utilisation du futur produit par le client}

Nous avons besoin de connaître quel est la valeur réelle du produit par rapport aux attentes des futurs clients. Un outil appelé LRI (Learning Revelancy Indicator, Indicateur de performance en apprentissage) a été développé. Il peut être utilisé en double sens. En effet, il peut être utilisé dans le but de mieux définir les attentes des futurs clients directs ou indirects en fonction de leur niveau ou de leur parcours. Il peut être envisagé également comme une analyse du produit proposé et vérifier qu'il est bien en conformité avec les attentes de ces mêmes futurs clients directs ou indirects. Nous appelons clients directs ou indirects; les clients qui vont utiliser le produit pour transmettre (clients directs) et les clients qui vont être confrontés au produit lors d'un apprentissage (clients indirects).

\subsubsection{La génération de la connaissance au cours du cycle de vie du produit étendu}

Un produit orienté pour la transmission et/ou l'apprentissage est un produit étendu contenant en lui même non seulement le produit matériel mais également des services associés. Dans notre cas, afin de simplifier l'étude, nous considérons le produit matériel avec un service associé : l'apprentissage. On suppose que des éléments de connaissance se sont accumulés (représentés par « $\mathrm{K} \Delta »)$. Il sera possible de produire des compétences nouvelles (représentées par « $\mathrm{C} \Delta »)$ pour 
les clients indirects. La différence entre la connaissance et la compétence dans son processus de transformation est basée sur les travaux de Giordan [10] et son modèle allostérique. (Allostérique est un terme issu du nom de certains organismes vivants -allostéries- aillant la particularité de ne se développer qu'en fonction des interactions de leur environnement proche).

\subsubsection{L'échelle comparative des moyens internes et externes}

Cette échelle comparative interne et externe n'est pas encore développée. C'est une comparaison entre trois facteurs : les ressources humaines internes et les ressources humaines externes potentielles, les ressources outils internes et les ressources outils externes, la potentialité de connaissance interne et la potentialité de connaissance externe.

\subsubsection{La chaîne de valeur ajoutée}

La partie inférieure de la grille utilise le modèle de la valeur ajoutée développé par Porter. Les éléments principaux résident dans le fait que les fonctionnalités principales et les supports du processus de développement du nouveau produit sont présents simultanément. Cette partie de la grille récapitule le résultat d'une analyse stratégique de la formation au niveau de chaque fonctionnalité primaire. L'analyse permet une analyse comparative de la situation dans divers secteurs de l'entreprise. Nous collectons toutes les informations au sujet des ressources humaines (compétences et ressources), outils (outils, procédés et infrastructures) et les connaissances potentiellement produites dans les différents services. L'objectif est de recenser rapidement ce que l'entreprise sera en mesure d'effectuer en interne et ce qu'elle sera obligée de sous traiter. 


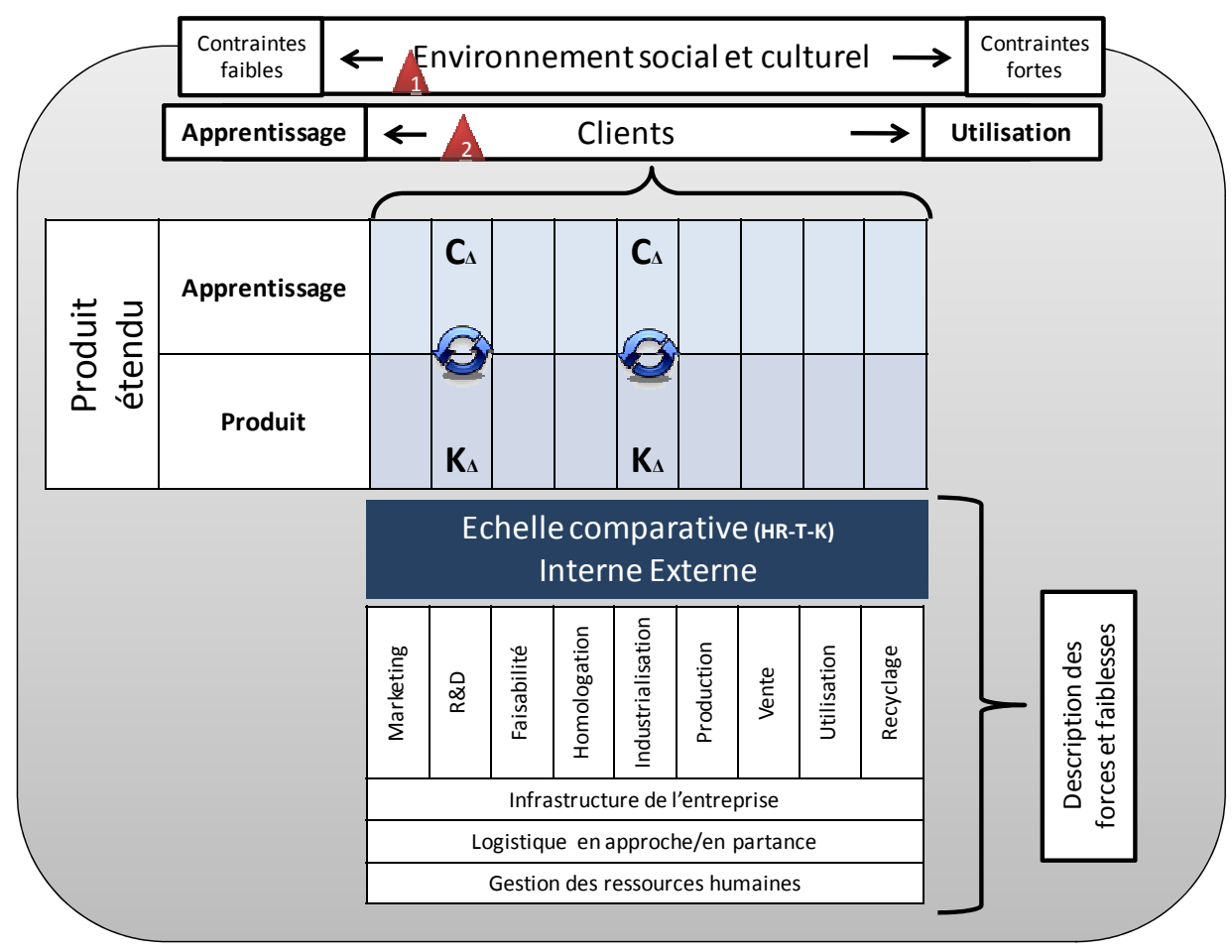

Figure 5 : Macro modèle du processus d'ingénition

\subsection{Les Micro MOdeles du Processus d'Ingenition}

Le but principal du processus d'ingénition est de se concentrer sur les compétences et la manière dont ces compétences notées « $C_{\Delta}$ » sont obtenues à partir des connaissances « $K_{\Delta} »$. Les compétences sont des éléments de la connaissance recueillis, structurés et utilisables par des usagers. Elles sont obtenues pendant les diverses séquences de formation. Formellement nous pouvons modéliser cette interaction principale par $K_{\Delta} \stackrel{i}{\longrightarrow} \mid C_{\Delta}$. La ligne verticale représente des séquences de formation. Le processus d'ingénition permet la structuration et l'observation de ce processus de transformation. La transformation de la connaissance en compétences utilise des supports particuliers. Un ou plusieurs objets d'apprentissage «learning object» (LO) peuvent devenir support de ce procédé, modélisé par : $K_{\Delta} \stackrel{i,\{L O\}}{\longrightarrow} \mid C_{\Delta}$. Par exemple, un progiciel mis en application dans un robot et le robot lui-même représentent les deux « learning object » pour un but d'apprentissage particulier. D'un point de vue générique, il est possible de décomposer « $K_{\Delta}$ » en une séquence d'éléments de connaissance plus détaillée. Le schéma du côté gauche de la Figure 6 représente cette séquence. « $\mathrm{Ci}$ et $\mathrm{Cf}$ » correspondent respectivement au niveau initial 
et final de la compétence des clients indirects. « $C_{\Delta}$ » correspond à la différence entre ces deux niveaux de compétence. Dans l'ellipse, nous pouvons voir le graphe des éléments interdépendants de la connaissance. L'ellipse modélise la frontière du processus d'ingénition. Chaque couple d'éléments de connaissance est connecté par un lien de dépendance. Par exemple, «K1 » est un élément nécessaire à la connaissance « K2 » (la base de l'électricité, « K1 », est exigée pour la compréhension du transistor, «K2»). Ce graphe permet de montrer ce que nous devons transmettre comme connaissance aux futurs stagiaires/concepteurs.

L'analyse du graphe des connaissances est basée sur les diverses dépendances recensées. Trois rapports logiques fondamentaux de dépendance ont été recensés :

Antécédence : La compréhension et la description de « $\mathrm{Kj} »$ ne sont pas possibles sans l'explication de « $\mathrm{Ki} »$. Dans ce cas-ci, « $\mathrm{Ki} »$ est un antécédent de « $\mathrm{Kj} »$.

Parallélisme : La compréhension de « $\mathrm{Ki} »$ et de « $\mathrm{K} \mathrm{j}$ » est indépendante. « $\mathrm{Ki}$ et $\mathrm{Kj}$ » peuvent être expliquées indépendamment (loi d'ohm et loi de Newton).

Simultanéité : « K1 et K2 » devront être traitées en même temps. Toutes les propriétés (plusieurs éléments : transitivité, commutativité, ...) de Boole devront être enseignées simultanément.

La considération de ces diverses dépendances pourrait définir directement la manière dont divers objectifs d'apprentissage seront utilisés (situation As Is). En analysant ces scénarii, on pourra alors : viser les compétences d'une situation d'apprentissage en montrant clairement les objectifs et leurs connexions, prendre des décisions stratégiques sur l'apprentissage et il devrait être possible d'établir des solutions de rechange ou alternatives en cas d'insuccès comme le montre la partie droite du schéma (Figure 6, situation To Be).

Chaque alternative d'apprentissage définit : des cibles de compétences, des objectifs d'apprentissage nécessaires, et le graphe des dépendances de la connaissance. La définition de ces solutions de rechange impose des contraintes structurelles et fonctionnelles sur la conception et la réalisation des objectifs d'apprentissage.

Le graphe de dépendance des connaissances ainsi obtenu se base sur l'analyse de la « compétence objectif », « Cf », s'appelle le graphe primaire de dépendance. Basés sur le choix des stratégies d'apprentissage, des graphes complémentaires peuvent être ajoutés aux graphes primaires afin de permettre aux clients indirects d'atteindre leurs «compétences objectifs» «Cf». Ces graphes 
complémentaires s'appellent graphes auxiliaires, leurs complexités peuvent donner un signe clair de l'efficacité de la stratégie choisie. L'utilisation d'un moteur pour montrer une liaison pivot n'est peut être pas la meilleure stratégie apprenante. Dans le chapitre 4, ces deux types de graphes seront illustrés plus en détail.
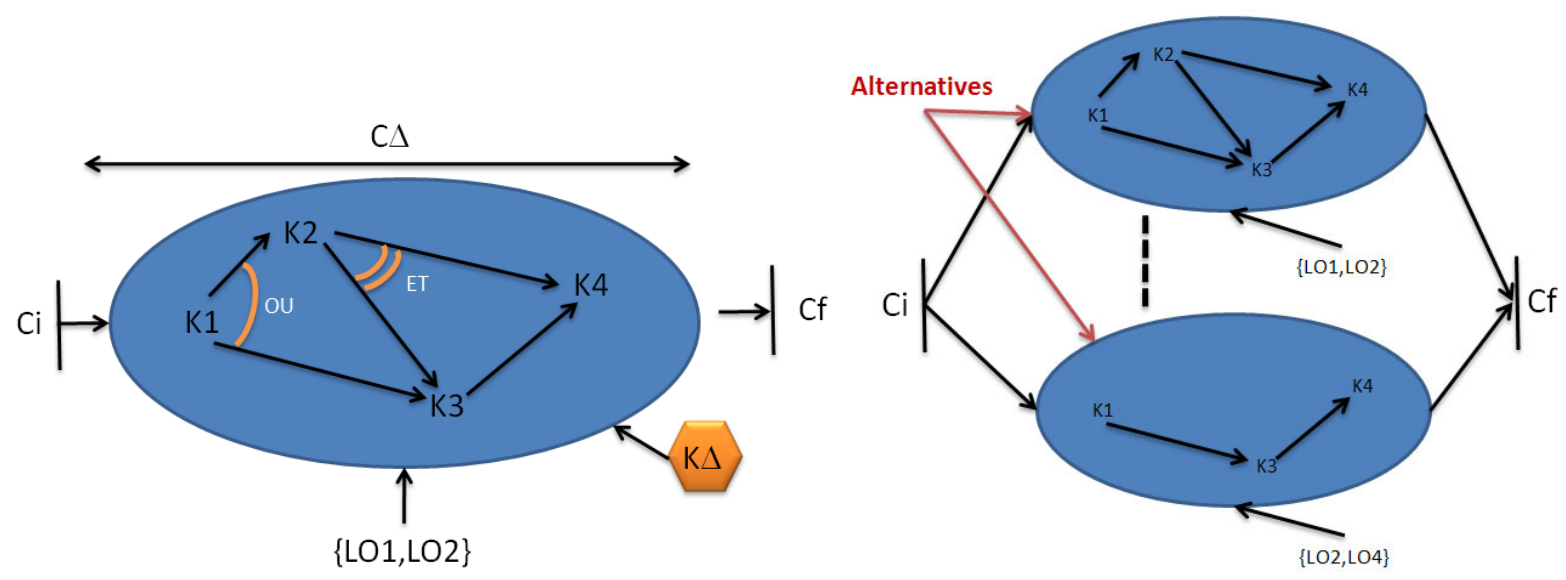

Figure 6 : Graphe des dépendances des connaissances

Pour compléter la Figure 6, il convient de rappeler quelques éléments. « $\mathrm{Ci}$ » est la compétence initiale de l'individu. Il passe par une décomposition simple de connaissances « $\mathrm{K} »$ organisées entre elles pour arriver à une compétence finale « Cf ». Par exemple, « $\mathrm{K} 1$ et $\mathrm{K} 2$ » peuvent être envisagées comme des pré-requis à l'élaboration de « K4 ».

\section{DESCRIPTION D'UN CAS ETUDIE}

Ce modèle est appliqué à une compagnie française appelée Innovia. Innovia conçoit et fabrique des produits pour les lycées et l'Université. Les dirigeants d'Innovia recherchent un nouveau produit comme support de formation dans les lycées, plus particulièrement dans les classes ayant des référentiels faisant appel aux principes des liaisons mécanique simples et complexes. Le produit est une bicyclette. L'environnement culturel et social n'impose pas de contraintes trop fortes au produit (voir le curseur 1 en haut de la Figure 5). C'est la raison pour laquelle le curseur est positionné à l'extrémité gauche de l'échelle. Un autre curseur (curseur 2 sur la Figure 5) est positionné sur l'échelle d'usage/apprentissage. Innovia sélectionne un produit destiné à être un support d'apprentissage. Le curseur est aussi sur la gauche. Les implications directes de ces deux échelles sont en termes de forme, matériaux, manœuvrabilité, etc., et devraient être prises en compte dès le début du projet de développement du produit. 
Les micro-modèles ont été développés pour représenter des alternatives potentielles pour une liaison pivot (K11) et une liaison pivot glissant (K12). On peut transmettre les connaissances pour chaque type de liaison par les approches suivantes : schéma cinématique 2D (K111 ou K121), schéma cinématique 3D (K112 ou K122) ou par l'expression théorique des torseurs (K113 ou K123). En représentant tous les éléments de la connaissance, le graphe primaire de dépendance est obtenu et montré dans les ellipses. Trois possibilités peuvent être utilisées pour illustrer une liaison pivot : un frein de bicyclette, un motoréducteur ou un moteur (ellipses Figure 7). Le graphe primaire est le même pour ces trois solutions cependant, en utilisant le moteur, il est clair que les éléments supplémentaires de connaissance devront être nécessaires dans le graphe de dépendance des connaissances. Le graphe auxiliaire contient : la théorie de la transmission de puissance (K4) dont nous aurons besoin pour la bonne compréhension des concepts fondamentaux de mécanique tels que l'énergie, la force, la puissance et le travail. Le graphe auxiliaire est représenté par les nœuds bleus et les liens pointillés.

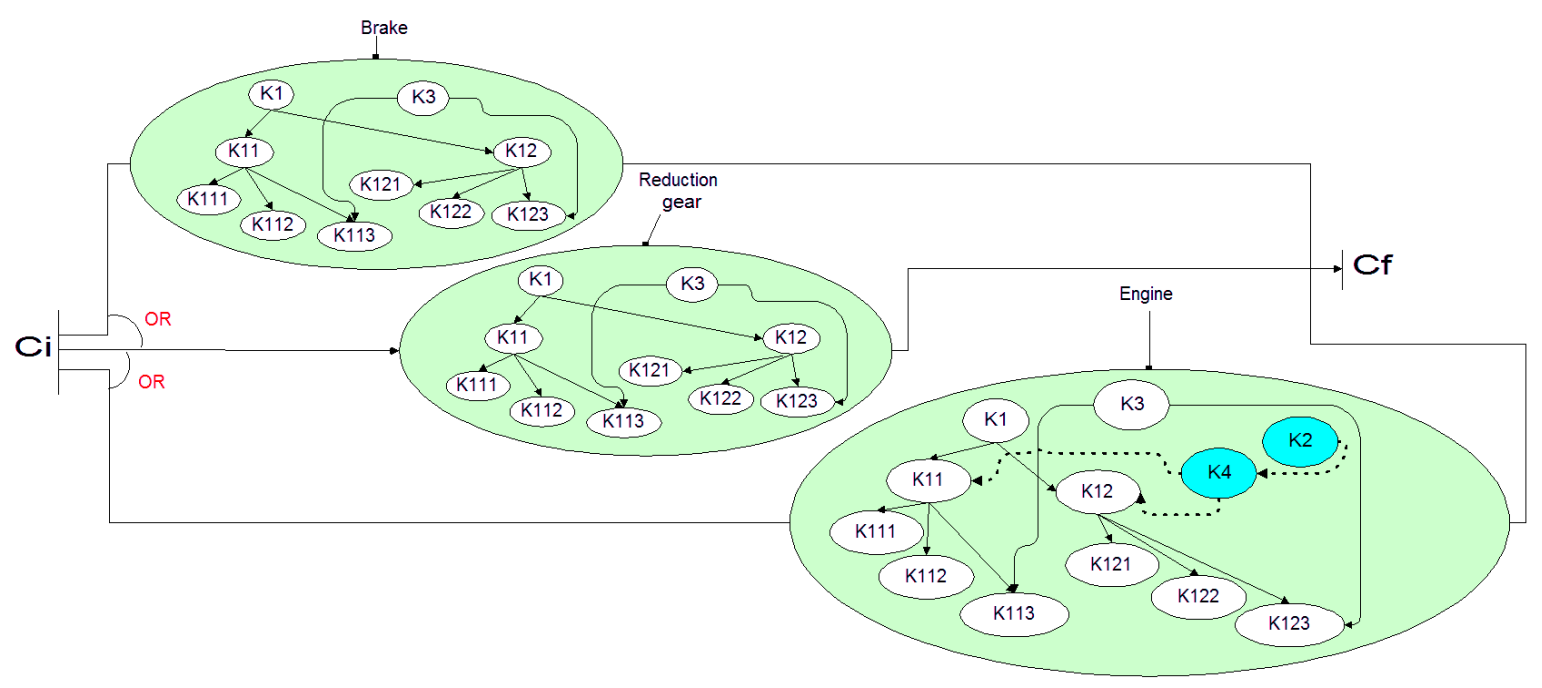

\begin{tabular}{|l|l|l|l|}
\hline K1 & $\begin{array}{l}\text { Mobilité d'un solide dans } \\
\text { l'espace }\end{array}$ & \multicolumn{2}{l|}{} \\
\hline K11 & Liaison Pivot & K12 & Pivot glissant \\
\hline K111 & Cinématique 2D & K121 & Cinématique 2D \\
\hline K112 & Cinématique 3D & K122 & Cinématique 3D \\
\hline K113 & Torseur & K123 & Torseur \\
\hline K2 & Puissance, Energie, Travail, & K3 & Manipulation de torseur \\
\hline
\end{tabular}

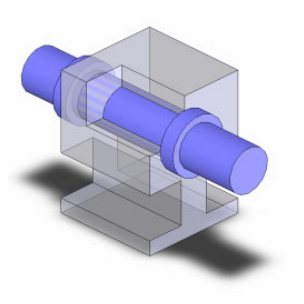




\begin{tabular}{|l|l|l|l|}
\hline & Force & & \\
\hline K4 & Transmission de puissance & \\
\hline
\end{tabular}

Figure 7 : Micro modèle d'une liaison pivot

Quelques observations à partir de ces micro-modèles:

- Graphe primaire. Il est nécessaire de montrer clairement le graphe primaire de dépendance des connaissances. Une analyse de ce graphe aide à extraire des recommandations pour les concepteurs et également pour les clients directs (formateurs). En faisant ainsi, nous sommes en train de faire de l'ingénierie de séquences d'apprentissage.

- Graphe auxiliaire. Dans cet exemple, on peut remarquer très facilement que l'utilisation d'un moteur pour la transmission des connaissances envisagées n'est pas très adaptée. C'est un indicateur important d'efficacité pour les utilisateurs finaux.

- En faisant l'extraction des graphes primaires et auxiliaires, de nouveaux objets d'apprentissage voient le jour.

- Il est possible d'analyser diverses solutions ou alternatives dans une stratégie d'estimation d'efficacité afin de choisir l'objet d'apprentissage le plus approprié à l'objectif attendu par les clients directs. Cela signifie que le motoréducteur pourrait représenter une meilleure solution qu'un frein de bicyclette dans d'autres situations d'apprentissage. D'où l'importance des choix initiaux stratégiques.

\section{CONCLUSION}

Dans cet article, nous avons étudié la « potentialité apprenante » d'un produit. Il est montré que la génération de la connaissance ne représente pas seulement une source interne importante d'innovation mais également que l'entreprise peut utiliser cette connaissance produite comme outil de positionnement de la société sur un marché concurrentiel. Après un bref état de l'art, nous avons proposé deux modèles issus du processus d'ingénition. Cette méthode poursuit deux objectifs : analyser et concevoir des produits pour transmettre et/ou apprendre, et analyser et concevoir des séquences d'apprentissage en vue d'améliorer les performances des acteurs de la conception.

En résumé, les principaux outils présentés dans la grille permettent : 
- de modéliser l'environnement social et culturel de l'entreprise dans un contexte d'apprentissage.

- de définir de mieux en mieux un produit dédié à l'apprentissage ou à son utilisation ou quelque chose entre les deux.

- de garder la trace de la génération de cette connaissance au cours de toutes les activités considérées.

- de mesurer les variations entre ce que l'entreprise peut faire elle même et ce qu'elle doit sous traiter.

Les micro-modèles représentent des graphes de dépendance de la connaissance. Ces graphes devraient correspondre à une sorte de « roadmap » nécessaire à la connaissance pour un but fixé stratégiquement. Chaque graphe a pour support un ou plusieurs objets d'apprentissage.

Notre recherche est concentrée sur un marché très dynamique : Le marché de l'éducation. Selon l'OCDE [OCDE, 1998], la dépense mondiale des systèmes éducatifs dans la décennie à venir représente quelque chose comme $2000 \mathrm{~b} \$$. Mais malheureusement des produits sont conçus et souvent industrialisés sans utiliser une approche scientifique spécifique. Nous nous sommes rendus compte qu'une somme considérable de travaux doit encore être effectuée dans ce domaine. L'approche a été expérimentée en partie dans une des plus grandes entreprises allemandes du secteur de l'éducation et a permis de stopper un projet de développement de produits! Les auteurs travaillent actuellement sur une description complète de la méthodologie d'ingénition et l'appliquent dans le système éducatif français. Les résultats de cette étude consolideront l'approche du processus d'ingénition.

\section{REFERENCES}

[1] Blessing L.T.M., A process-based approach to computer-supported engineering design, $\mathrm{PhD}$ Thesis, University of Twente, Enschede, The Netherlands, 1994.

[2] Caulier P., Méthodologie de capitalisation et de réutilisation de connaissances pour l'aide à la supervision des procédés automatisés complexes: application à la supervision du trafic téléphonique de l'Ile de France, Thèse de doctorat, Université de Valenciennes et du HainautCambresis, France, 1997.

[3] Cross N., Dorst K., Roozenburg N., Research in Design Thinking, Delft University Press, 1992. 
[4] de Hoog R., Benus B., Metselaar C., Vogler M., The common KADS organisation model, 1993.

[5] Dixon J., On Research Methodology Towards a Scientific Theory of Engineering Design, Artificial Intelligence for Engineering Design Analysis and Manufacturing (AI-EDAM), Academic Press, Vol.1, n³, pp.145-156, 1987.

[6] Ermine J.L., Chaillot M., Bigeon P., Charreton B., Malavieille D., MKSM, Méthode pour la gestion des connaissances, Ingénierie des systèmes d'information, AFCET-Hermès, Vol.4, $\mathrm{n}^{\circ} 4$, pp.541-575, 1996.

[7] Ermine J.L., Les processus de la gestion des connaissances, Extraction et gestion des connaissances, H. Briand, F. Guillet (éd.), Hermès, 2001.

[8] Ermine J.L., Génie logiciel et génie cognitif pour les systèmes à base de connaissances, collection Tec et Doc, Lavoisier, Paris, 1993.

[9] Evbuomwan N.F., Sivaloganathan S., Jebb J., A survey of Design Philosophies, Models, Methods and Systems, Journal of Engineering Manufacture, Vol.210, pp.301-320, 1996.

[10] Giordan A., Girault Y. , Clement P., "Conceptions et connaissance” (in french), Peter Lang, 1994.

[11] Glaser N., Contribution à l'acquisition et à la modélisation des connaissances dans un cadre multi-agents, Thèse de Doctorat, Université Henri Poincaré - Nancy I, 1996.

[12] Le Moigne J.L., La théorie du système général - Théorie de la modélisation, Presses Universitaires de France, Paris, 1977.

[13] Le Moigne J.L., La modélisation des systèmes complexes, Afcet Systèmes Ed. Dunod, Paris, March L., The Logic of Design, Development in design Methodology, Cross N. (éd.), John Wiley \& Sons, 1984.

[14] Matta N., Ermine J.L., Gestion des connaissances et conception mécanique, Journée AIPPriméca "Dynamique des connaissances en conception: acquisition, capitalisation et réutilisation », Grenoble, France 22 mai 2001.

[15] Merlo C., "Modélisation des connaissances en conduite de l'ingénierie: Mise en æuvre d'un environnement d'assistance aux acteurs ", Thèse de l'Université Bordeaux 1, décembre 2003. 
[16] Nonaka, Toyama and Konno: SECI, "Ba and Leadership: a Unified Model of Dynamic Knowledge Creation”, Long Range Planning 33, pp 5-34, 2000.

[17] Pahl G., Beitz W., Engineering design, London, Design Council, 1984.

[18] Perrin J., Villeval M.C., Lecler Y., Les requis organisationnels et institutionnels pour développer la coopération au sein des activités de conception, Communicationnel pour Concevoir, Caelen J., Zreik K. (éds), Europia Productions, Paris, 1995.

[19] Perrin J., Concevoir l'innovation industrielle, méthodologie de conception de l'innovation, CNRS éditions, Paris, 2001.

[20] Porter M.E., "The Competitive Advantage of Nations", Harvard Business, Review 90211, March 1990.

[21] Roozenburg N.F., Eeckels J., Product Design : Fundamentals and Methods, John Wiley \& Sons, 1995.

[22] Schön D., Apprentissage organisationnel et épistémologie de la pratique, Les Limites de la rationalité, Reynaud B. (éd.), La Découverte, Paris, t.2, 1997.

[23] Schreiber G., Breuker J., Biedeweg B., Wielinga B.J., Modelling in KBS development, Avignon, $1: 283-296,1988$.

[24] Schreiber G., Wielinga B., Akkermans H., van de Velde W., Anjewierden A., CML: the CommonKADS Conceptual Modelling Language, Proceedings of EKAW'94, Hoegaarden, Belgium, Steels \& al, eds, Springer-Verlag, pp.1-25, 1994.

[25] Simon G., Modèles et méthodes pour la conception des mémoires d'entreprise - Le système DOLMEN : une application en métallurgie, Thèse de doctorat, Nancy 1997.

[26] Wielinga B.J., Van de Velde W., Schreiber G., Akkermans H., Expertise model definition document, Research model KADS II/M2/UvA/026/1.1, ESPRIT Project P5248 KADS II, KADS II Consortium, 1993. 